Meta

Journal des traducteurs

Translators' Journal

\title{
La traduction au Canada de 1791 à 1867
}

\section{Jacques Gouin}

Volume 22, numéro 1, mars 1977

Histoire de la traduction au Canada

URI : https://id.erudit.org/iderudit/002954ar

DOI : https://doi.org/10.7202/002954ar

Aller au sommaire du numéro

Éditeur(s)

Les Presses de l'Université de Montréal

ISSN

0026-0452 (imprimé)

1492-1421 (numérique)

Découvrir la revue

Citer cet article

Gouin, J. (1977). La traduction au Canada de 1791 à 1867. Meta, 22(1), 26-32.

https://doi.org/10.7202/002954ar d'utilisation que vous pouvez consulter en ligne.

https://apropos.erudit.org/fr/usagers/politique-dutilisation/ 


\section{La traduction au Canada de 1791 à 1867}

Il y a une dizaine d'années, alors que je caressais le rêve d'écrire une histoire de la traduction au Canada depuis Jacques Cartier, je ne me doutais pas que l'idée était déjà dans l'air et que d'autres, en même temps que moi, songeaient à un projet similaire. Par la suite, deux collègues, MM. Paul Horguelin et Jean Delisle, m'ayant fait part de leurs intentions, je renonçai à mon projet qui, du reste, était trop ambitieux pour un seul homme, et acceptai volontiers de coordonner mes recherches avec les leurs, en vue d'une histoire collective, devenue l'objet de ce numéro spécial de Meta.

Au début de mes recherches, j'avais été frappé par l'absence de toute histoire méthodique et complète de la traduction, pourtant l'une des plus anciennes professions du monde. $\grave{A}$ plus forte raison, me disais-je, chcrcherions-nous en vain une histoire de la traduction au Canada. Bien sûr, il existe de nombreux ouvrages qui traitent de traduction, mais tous ne parlent de son histoire que de façon fragmentaire ou indirecte. Pourtant, me disais-je encore, il n'est pas exagéré d'affirmer que la traduction - tout comme l'interprétation, profession jumelle - ne cesse de s'exercer, avec plus ou moins de bonheur ou de compétence, il est vrai, depuis les débuts mêmes de notre histoire. Aussi, convient-il de saluer avec joie ce premier effort collectif de la revue Meta, visant à doter nos collègues d'une amorce d'histoire de leur profession.

Un historien plutôt hargneux à l'égard des conséquences désastreuses de 1760, a écrit : "Si la Conquête a fermé plusieurs canaux de promotion aux Canadiens, il faut admettre qu'elle leur a ouvert une nouvelle carrière : celle de la traduction ${ }^{1}$. \Nous traduisons, en effet, depuis la Conquête, et peut-être plus qu'aucun autre peuple au monde. Et des élites successives ont consacré leur vie à cette activité ingrate. Dès 1791 , on constate, par exemple, que notre ancienne classe seigneuriale, d'exclusivement militaire et commerciale qu'elle avait été jusque-là, se voit graduellement évincée (sauf de rares exceptions, comme les de Salaberry et certains de Lanaudière) par les Britanniques, pour échouer dans le seul fonctionnarisme, et à des postes de second plan ${ }^{2}$.

1. Michel Brunet, les Canadiens après la Conquête (1759-1775), tome I : De la Révolution canadienne de la Révolution américaine, Montréal, Fides, 1969 , p. 24-25.

2. Fernand Ouellet, Histoire économique et sociale du Québec (1760-1850), Montréal et Paris, Fides, 1966, notamment les chapitres sur le déclin de la classe seigneuriale. 
De 1794 à 1812, si l'on peut classer en six catégories les postes occupés par les Britanniques et les seigneurs canadiens, on voit que le "traducteur français \& du Gouverneur occupe la quatrième catégorie avec les greffiers, secrétaires, etc., juste avant les commis de bureau, les simples traducteurs et interprètes, et les interprètes de cour. Or, tous ces postes de traducteurs et interprètes sont occupés, à l'époque, par des membres de grandes familles seigneuriales plus ou moins déchues. On relève notamment les noms de Xavier de Lanaudière, traducteur et secrétaire français de la province, dont le frère Charles était aide-de-camp de lord Dorchester, et C.-E. de Léry, assistant-greffier et traducteur du Conseil législatif ${ }^{3}$. Et, quand Xavier de Lanaudière mourra, le 15 avril 1813, lui succédera à son poste nul autre que Philippe-Aubert de Gaspé 4 , autre seigneur déchu, qui se réfugiera bientôt dans ses souvenirs nostalgiques d'un monde évanoui. $\grave{A}$ propos de ce déclin marqué de l'ancienne classe seigneuriale, écartée à tout jamais non. seulement du grand commerce, de l'industrie et des affaires publiques, mais aussi des grandes charges de l'État - déclin qui n'est pas sans évoquer le sort analogue réservé à l'ancienne noblesse française après 1789 qui, elle, fut déplacée par une nouvelle classe sociale et non par une autre «nation » - citons la conclusion de deux jeunes historiens québécois contemporains : «[De 1794 à 1812] ... les Canadiens français remplissent surtout des fonction de traducteurs, de greffiers, d'interprètes, etc., hormis pour trois ou quatre conseillers exécutifs. Ils font le pont entre les services de l'État et la population ${ }^{5}$. $\gg$ En 1810, par exemple, le bureau du secrétariat de la province du Bas-Canada et du gouverneur ne compte que des Britanniques, sauf pour le traducteur des proclamations ${ }^{\circ}$. Veut-on une autre preuve, froidement statistique cette fois, du déclin irréversible de l'ancienne classe dirigeante du Québec au cours des cinquante premières années consécutives à 1760 , et de son orientation marquée vers la traduction et l'interprétation? En 1804, la fameuse Compagnie du Nord-Ouest, rivale de la Hudson Bay Company dans le commerce des fourrures, comptait 43 actionnaires anglophones contre 2 francophones - ceux-ci par ailleurs de la classe seigneuriale, bien entendu - alors qu'elle comptait 56 interprètes francophones contre 12 anglophones. Ces seuls chiffres sont révélateurs de l'orientation désormais imprimée à la collectivité québécoise ?.

Un dernier exemple de la dégringolade sociale de l'élite québécoise au cours des années 1791-1812 serait peut-être celui du lieutenant-colonel Charles-Pierre Frémont, quartier-maître général adjoint des milices du Bas-Canada, qui joua un rôle important dans la bataille de Châteauguay en 1813 , et qui, à sa mort en 1827, était traducteur et greffier des bills privés. Petit-fils de Pierre Boucher de Boucherville - dont le long mémoire à Louis XIV sur l'état de la NouvelleFrance en 1663 est resté justement célèbre - il était l'oncle de John-Charles

3. Gilles Paquet et Jean-Pierre Wallot, $<$ La liste civile du Bas-Canada (1794-1812) : essai d'économie politique $*$, dans Revue d'histoire de l'Amérique française, vol. $24, \mathrm{n}^{\circ} \mathrm{1}$, juin 1970 , p. $17-19$.

4. G.-A. Drolet, Zouaviana, appendice intitulé * Les Cahiers de mon arrière-grand-père (1778-1828) \, Montréal, Eusebe Sénécal \& Cie, 1898, p. 580.

6. Paquet et Wallot, op. cit., p. 20.

6. Ibid.

7. L.-R. Masson, les Bourgeois de la Compagnie du Nord-Ouest : Récits de voyages, lettres 
Frémont, qui connut une fulgurante carrière aux États-Unis. L'un des plus grands explorateurs de l'Ouest américain, John-Charles devint en effet général d'armée pendant la Guerre de Sécession, puis candidat à la présidence des Etats-Unis ${ }^{8}$. Etrange retour des choses, cependant : Charles-Pierre Frémont fut également l'arrière-grand-père du major-général Édouard de Bellefeuille Panet, qui servit avec distinction pendant les deux guerres mondiales, et qui vit toujours retiré à Montréal, ayant atteint près de 95 ans ${ }^{9}$.

$\mathrm{Si}$, de 1791 à 1812, la traduction officielle dans le Bas-Canada est assurée par une certaine élite, devenue, par la force des choses, intellectuelle, après avoir été industrielle, commerciale, politique et militaire, il convient maintenant de se demander quelle était la qualité de cette traduction. Dès le 6 février 1769, le texte français des proclamations porte désormais la mention : "Traduit par ordre de Son Excellence, F.-J. [François-Joseph] Cugnet, S.F. [Secrétaire français] ${ }^{10}$. Pendant vingt ans, soit jusqu'à sa mort en 1789, François-Joseph Cugnet, premier traducteur officiel du Canada, assura tant bien que mal toute la traduction qui devait se faire au niveau officiel. Cugnet, né Canadien, et l'un des avocats les plus versés de son temps en droit français, fit une besogne * traductionnelle » assez honorable ${ }^{11}$. Son père, François-Étienne, avait été un homme d'affaires fort entreprenant ${ }^{12}$. Son fils, Jacques-François, avocat comme son père, puis associé et successeur de ce dernier dans la charge de traducteur, manifeste déjà les faiblesses de traduction qui dépareront nos textes officiels pendant un demi-siècle. Déjà, dès 1780 , les traductions de Cugnet père étaient devenues « franchement mauvaises ${ }^{13} \gg$. Sans doute devenait-il surchargé de travail, puisqu'il demandait et obtenait bientôt de l'aide, d'abord celle de son fils, puis de Pierre-Amable de Bonne, autre seigneur, futur député en 1792, puis juge et, enfin, membre du Conseil exécutif ${ }^{14}$. Mais, après la mort de Cugnet père en 1789 , il semble que le fils ne fît que se détériorer, non seulement dans sa vie privée, mais surtout dans ses traductions. Dans les dernières proclamations qui précédèrent la division de la province en Bas et Haut-Canada, on commence à relever en effet les « horreurs * qui ne cesseront, pendant cinquante ans, de truffer nos traductions officielles. Signalons : «La provision spéciale faite pour le soutien des juges... \ (The special provision made for the support of the judges...) ; «allouer des honoraires 》 (to allow fees) ; « examen d'un témoin 》 pour « interrogatoire»; " retour d'un writ d'appel $\gg$ (return of a writ of appeal).

et rapports inédits relatifs au nord-ouest canadien, publiés avec une esquisse historique et des annotations ; première publication en 1889-1890; réimpression en deux volumes, en 1960, par Antiquarian Press Ltd., New York, vol. I, p. 395-413.

8. Pierre-Georges Roy, la Famille Frémont, Lévis, (s.é.), 1902, p. 9.

9. Pierre-Georges Roy, la Famille Panet, Lévis, (s.é.), 1906, p. 113 ; aussi, Lucien Brault et Jacques Gouin, les Panet : Histoire d'une lignée militaire canadienne (1740-1975), chap. V, à paraître en 1977, sous les auspices du Musée canadien de la Guerre, Ottawa.

10. Pierre Daviault, Traducteurs et traduction au Canada, Mémoires de la Société royale du Canada, Section I, 1944, p. 81.

11. Pour une biographie détaillée de ce premier traducteur officiel canadien, voir Marine Leland, * François-Joseph Cugnet $(1720-1789)$, série de treize articles, parus dans la Revue de l'Université Laval, vol. 16 à 19, 1961-1964.

12. Cameron Nish, François-Etienne Cugnet 1719-1751 : Entrepreneur et entreprises en Nouvelle-France, Montréal, Fides, 1975,185 p.

13. Daviault, op. cit., p. 84 .

14. Ibid 
Au début, c'est-à-dire de 1764 à 1789 , la traduction officielle était excellente. Après 1789 , et cela jusque vers les années 1850 , la traduction se détériore, sans doute parce qu'elle est confiée de plus en plus à des gens de formation anglaise. On n'ignore pas, en effet, que bon nombre d'anciens seigneurs, devenus traducteurs, s'étaient également anglicisés, encore une fois par la force des choses. De plus, comme on s'éloignait constamment des sources françaises, on s'enfonçait dans une atmosphère exclusivement anglaise. Les institutions britanniques se faisaient sentir de plus en plus. Pour participer le moindrement à la vie publique, il fallait s'en imprégner. La France était de plus en plus éloignée; on ne s'en rapprochera qu'en 1855, avec la venue de la Capricieuse au Canada. Dans une étude que Pierre Daviault devait ajouter à ses premières notes sur la traduction depuis 1764 , on devait voir «... à quel niveau de déchéance finit par tomber le français de nos traductions officielles, les horreurs qui s'écrivaient vers le milieu du dix-neuvième siècles ${ }^{15}$ ». Mais, il concluait :

Nous constaterons aussi qu'en dépit de tout, une lueur persistait, de sorte que, lorsque reprirent les relations avec la France [en 1855], les améliorations commencèrent à se manifester. Si bien que, de nos jours [1944], du moins en certains coins où l'on se soucie de la pureté de la langue, s'écrit un français de traduction infiniment supérieur à celui de la période noire, c'està-dire le $19^{\mathrm{e}}$ siècle ${ }^{16}$.

S'il entrevoyait cet espoir de jours meilleurs pour la traduction au Canada, c'est que Pierre Daviault avait été à l'école de ce grand maître que fut Léon Gérin, fils d'Antoine Gérin-Lajoie, avec qui, sous le gouvernement de l'Union, la traduction avait commencé à s'améliorer.

En effet, de 1850 à 1867 , un seul nom semble dominer le monde de la traduction au Canada, et c'est celui d'Antoine Gérin-Lajoie. L'auteur de Jean Rivard et d'autres remarquables travaux d'histoire et d'érudition, naquit en 1824. Dès l'âge de vingt ans, ses études à peine terminées, il se rendait aux États-Unis pour y apprendre l'anglais. Il n'y resta que dix-sept jours, après maintes tribulations. En 1852, il se rend de nouveau aux États-Unis pour perfectionner son anglais. En novembre de la même année, il est nommé traducteur à l'Assemblée législative ${ }^{17}$. Sans s'en rendre compte, ce fut un tournant dans son existence jusque-là * errante et vagabonde \$. Pendant les dix prochaines années, il suivra le Parlement de Québec à Toronto, puis de Toronto à Québec, et enfin de là à Ottawa en 1865, à titre de bibliothécaire adjoint du Parlement. Dès le 4 novembre 1852, il avait terminé la traduction d'une série de documents relatifs à la tenure seigneuriale ${ }^{18}$. Le 26 janvier 1853, voici ce qu'il écrivait dans son journal :

Ma vie a été assez monotone depuis quelque temps. J'ai continué à traduire assidûment de neuf heures du matin à quatre heures et demie de l'aprèsmidi... Nous sommes six traducteurs : Guillaume Lévesque (chef), D.-P. Myrand, J. Huston, Wm Fanning, Frs Amiot... L'arrangement du travail dans

15. Daviault, op. cit., p. 86-87.

16. lbid., p. 87 .

17. Louvigny de Montigny, Antoine Gérin-Lajoie, Toronto, The Ryerson Press, Collection * Makers of Canadian Literature *, s.d., p. 13

18. LÉon Gérin, Antoine Gérin-Lajoie : la résurrection d'un patriote canadien, Edition du centenaire, Montréal, 1975, p. 94. 
le bureau des traducteurs me déplaît souverainement. Chacun travaille conme il le juge à propros. Un traducteur peut faire six fois plus d'ouvrage qu'un autre, et pourtant tous deux reçoivent le même salaire. Il y aurait plus de justice à nommer un chef responsable qui ferait entreprendre l'ouvrage et serait chargé de tout surveiller, et de fixer le prix des traductions.

J'ai eu l'occasion de voir depuis quelques mois combien il y a, en effet, de perte de temps, de gaspillage, parmi les employés publics. Il faudrait une réforme complète. Le favoritisme, le népotisme, sont en grande partie la cause ou la source de ces abus ${ }^{19}$.

Cet extrait du journal intime d'Antoine Gérin-Lajoie atteste, comme il fallait un peu s'y attendre, qu'il n'y a rien de bien nouveau sous le soleil... de la traduction. La « réforme complète » qu'il préconisait a proliféré, comme on le sait, sous la forme d'innombrables nouvelles "structures», à tel point qu'on dit souvent maintenant que la plus grave maladie dont souffre le Bureau des traductions d'Ottawa est la «structurite aiguë ». Mais, on y trouve aussi la mention de son chef, Guillaume Lévesque, dont la vie agitée mérite une digression. En 1838, ayant participé à l'opération de Napierville, sous les ordres de Robert Nelson, «Président de la République du Bas-Canada », Guillaume Lévesque avait été condamné à mort ; mais sa sentence avait été commuée pour l'exil en Australie avec 58 autres rebelles. Grâce à l'intervention de son oncle, Pierre-Louis Panet, auprès d'un autre de ses oncles, William von Moll Berczy, qui avait l'oreille de sir John Colborne, Guillaume échappa à l'exil en Australie, à condition de quitter le Canada ${ }^{20}$. Réfugié en France, il trouva un emploi au ministère des Affaires étrangères. En 1843, lorsque Louis-Amédée Papineau, fils du chef patriote, rencontra Guillaume en France, celui-ci vivait sous le nom d'emprunt de Ramezay d'Ailleboust, en souvenir de la seigneurie d'Ailleboust qui appartenait à la famille de sa mère. $\AA$ la suite de l'amnistie accordée aux rebelles de 1837-1838, Guillaume revint au Canada en 1843, fut appelé au Barreau du Québec, et par la suite se trouva aux côtés de Gérin-Lajoie à titre de traducteur parlementaire sous le gouvernement de l'Union. Guillaume Lévesque mourut célibataire, à Québec, le 6 janvier 1856, et fut inhumé dans le cimetière de la seigneurie de sa tante Louise-Amélie Panet von Moll Berczy, à Sainte-Mélanie d'Ailleboust ${ }^{21}$.

Le 24 octobre 1854, Antoine Gérin-Lajoie soumettait à M. Victor Sicotte, président de la Chambre, un «projet de réorganisation des bureaux de traduction de l'Assemblée législative ${ }^{22} \gg$. On lui confiait peu après d'importantes tâches de traduction, dont celle de la loi abolissant le régime seigneurial, la loi sur les réserves du clergé et la loi sur l'organisation des communes ou municipalités ${ }^{23}$. Son projet de réorganisation du personnel de traduction de la Chambre d'assemblée fut enfin accepté. Il y aura désormais trois bureaux : Guillaume Lévesque et lui, aux lois ; Myrand et Eugène-Philippe Dorion, aux documents ; et Fanning,

19. Léon Gérin, op. cit., p. 94-95.

20. Lucien Brault et Jacques Gouin, les Panet : Histoire d'une lignée militaire canadienne, (1740-1975), Appendice III : * La lignte montréalaise de la famille Panet *, à paraître en 1977, sous les auspices du Musée canadien de la Guerre, Ottawa.

21. Aegidius Fauteux, Patriotes de 1837-1838, Montreal, Editions des Dix, 1950, p. 302-303.

22. Iéon Gérin, op. cit, p. 104.

23. Ibid. 
aux votes et délibérations. Jusque vers les années 1925 , cette organisation du personnel de la traduction à la Chambre des communes ne devait pas être modifiée, sauf par l'adjonction (suite à la Confédération en 1867) d'un bureau pour la traduction des débats. Ce fut sans doute la structure qui persista jusqu'à la centralisation de 1934. «Cette réforme administrative, opérée sur son conseil et d'après ses indications, affirme son fils Léon Gérin, fut le dernier fleuron de sa carrière de traducteur ${ }^{24}$. $\gg$ Il deviendra peu après chargé de la section française de la bibliothèque du Parlement.

Comme on vient de le voir, Antoine Gérin-Lajoie fut la cheville ouvrière de la traduction officielle au Canada des années 1850 à 1860 . On pourrait signaler également ici que François-Xavier Garneau, son contemporain, fut traducteur avant d'être historien. De 1860 à 1870 , un autre éminent traducteur laissera son norn à l'histoire de la profession: Eugène-Philippe Dorion, déjà mentionné cidessus. Avocat et homme de lettres, Dorion fut appelé dès 1859 à diriger le bureau de traductions de l'Assemblée législative du Canada-Uni, poste qu'il occupa ensuite à Ottawa à la Chambre des communes, dès la Confédération. Ses contemporains ont vanté sa connaissance des langues mortes, de l'anglais, du français et de quelques langues indiennes. Il améliora sensiblement le texte français des lois de l'époque, mais il dut parfois s'incliner devant la volonté et le mauvais goût linguistique des hommes politiques, comme cela arrive encore parfois de nos jours ! On raconte en effet que c'est Georges-Étienne Cartier lui-même qui aurait imposé à Dorion la traduction du mot Dominion par Puissance, dans l'Acte de 1867. Eugène-Philippe Dorion mourut à Ottawa en 1872, après avoir été président de la Société Saint-Jean-Baptiste et de l'Institut canadien-français d'Ottawa ${ }^{25}$. Il faut mentionner qu'à cette même époque Louis Fréchette et Pamphile Le May, les deux plus célèbres poètes de leur temps, ont aussi été traducteurs à un moment ou l'autre de leur carrière. De Le May, on retiendra surtout sa traduction du célèbre roman de William Kirby, The Golden Dog, et surtout sa version en alexandrins de la non moins célèbre Évangéline de Longfellow. On pourrait enfin mentionner la traduction des romans de sa belle-sœur Rosanna Leprohon par Joseph-Édouard Lefebvre de Bellefeuille, seigneur des Mille-fles, retiré à Saint-Eustache, dont un petit-fils, Lionel, sera aussi traducteur.

Si l'on divise la période étudiée (1791-1867) en deux parties, on peut conclure que, de 1791 à 1850 environ, la traduction officielle accuse un réel déclin, par rapport à la période précédente de 1764 à 1791. Par ailleurs, de 1850 à 1867 , nous assistons à une nette amélioration de la profession, sans doute attribuable au réveil de la vie intellectuelle qui gravitait alors autour de l'École patriotique de Québec, sous l'égide des Crémazie, Casgrain, Garneau, Fréchette et, bien entendu, Gérin-Lajoie. Dès 1760, la traduction était devenue un mal nécessaire, seul moyen qui permît au Conquérant de communiquer ses volontés au Conquis. Peu à peu, cet expédient se transforma en profession. Mais, comme toute entreprise humaine, la traduction au Canada connut des hauts et des bas.

24. Léon Gérin, op. cit., p. 107.

25. Jean-Charles Bonenfant, Eugène-Philippe Dorion, biographie à paraître dans un prochain volume du Dictlonnaire biographique du Canada, lettre à l'auteur du présent article, 4 septembre 1970. 
Souhaitons, pour nos contemporains et successeurs, que notre époque marquera un sommet : nous le méritons bien, il me semble, après tant d'efforts déployés depuis bientôt quatre siècles et demi, c'est-à-dire depuis les deux premiers glossaires indiens-français de Jacques Cartier.

JACQUES GOUIN 